OPEN ACCESS

Edited by:

Jessica Lee Oliva,

Monash University, Australia

Reviewed by:

Alla B. Salmina,

Krasnoyarsk State Medical University

named after Prof.

V.F.Voino-Yasenetsky, Russia

Paolo Mongillo,

University of Padua, Italy

*Correspondence:

Evan L. MacLean

evanmaclean@email.arizona.edu

Specialty section:

This article was submitted to

Comparative Psychology,

a section of the journal

Frontiers in Psychology

Received: 08 May 2017 Accepted: 01 September 2017 Published: 20 September 2017

Citation

MacLean EL, Gesquiere LR, Gee NR, Levy K, Martin WL and Carter CS (2017) Effects of Affiliative Human-Animal Interaction on Dog

Salivary and Plasma Oxytocin and Vasopressin.

Front. Psychol. 8:1606.

doi: 10.3389/fpsyg.2017.01606

\section{Effects of Affiliative Human-Animal Interaction on Dog Salivary and Plasma Oxytocin and Vasopressin}

\author{
Evan L. MacLean ${ }^{*}$, Laurence R. Gesquiere ${ }^{2}$, Nancy R. Gee ${ }^{3,4}$, Kerinne Levy $^{5}$, \\ W. Lance Martin ${ }^{6}$ and C. Sue Carter ${ }^{7}$
}

${ }^{1}$ School of Anthropology, University of Arizona, Tucson, AZ, United States, ${ }^{2}$ Department of Biology, Duke University, Durham, NC, United States, ${ }^{3}$ WALTHAM Centre for Pet Nutrition, Leicestershire, United Kingdom, ${ }^{4}$ Department of Psychology, State University of New York, Fredonia, NY, United States, ${ }^{5}$ Canine Companions for Independence, Santa Rosa, CA, United States, ${ }^{6}$ Martin-Protean, LLC, Princeton, NJ, United States, ${ }^{7}$ Kinsey Institute and Department of Biology, Indiana University, Bloomington, IN, United States

Oxytocin (OT) and vasopressin (AVP) are neuropeptides with diverse effects on social behavior, cognition and stress responses. Recent studies suggest that OT facilitates and responds to affiliative forms of human-animal interaction (HAl). However, previous studies measuring OT and AVP in dogs have been limited to measures from blood or urine, which present concerns related to the invasiveness of sample collection, the potential for matrix interference in immunoassays, and whether samples can be collected at precise time points to assess event-linked endocrine responses. Previous studies from our laboratory validated salivary measures of OT and AVP in dogs, however, it is currently unknown whether these measures respond dynamically to aspects of HAl. Here, we investigated the effects of affiliative forms of HAI on both plasma and salivary OT and AVP in dogs. We employed a within- and between-subjects design with a group of Labrador retrievers and Labrador retriever $\times$ golden retriever crosses $(23$ females, 15 males). Half of the dogs engaged in 10 min of free-form friendly interaction with a human experimenter (HAl condition), and the other half rested quietly in the same environment, without human interaction (control condition). We collected blood and saliva samples before, and immediately following both experimental conditions, and all samples were analyzed using enzyme-linked immunosorbent assays (ELISAs) following previously validated protocols. Dogs participating in HAl exhibited a significant increase in both salivary OT (+39\%) and plasma OT (+5.7\%) whereas dogs in the control group did not. Salivary AVP showed no change in the HAl group but increased significantly $(+33 \%)$ in the control group. Plasma AVP decreased significantly following HAI (-13\%) but did not change across time in the control condition. Within the dogs exposed to HAl, increases in salivary OT, and decreases in plasma AVP, were predicted by the extent of affiliative behavior between the dog and human (indexed by scores from a principal components analysis of social behaviors between the dog and human). Collectively our results suggest that measures of salivary OT and AVP provide useful biomarkers in studies of HAl, and afford a flexible and non-invasive toolkit than can be employed in diverse research contexts.

Keywords: oxytocin, vasopressin, dog, human-animal interaction, ELISA, saliva, plasma 


\section{INTRODUCTION}

Studies throughout the last three decades have explored the psychological and physiological effects of human-animal interaction (HAI). Often the aim of such studies is to characterize the mechanisms through which non-human animals affect human health and wellbeing, and in turn, how interaction with humans affects animal participants. Recently such studies have begun to focus on the neuropeptide oxytocin (OT), which, together with (structurally related) arginine vasopressin, is well known for its roles in facilitating selective social bonds, and regulating various aspects of social behavior and cognition in mammals (Carter et al., 2008). For example, central OT administration can facilitate maternal behavior in sexually naïve rodents (Pedersen and Prange, 1979; Caldwell and Young, 2006), and both OT and AVP are critical to the formation of partner preference in pair-bonded species (Carter et al., 1992). With regard to the establishment of selective social attachment, the OT receptor (OXTR) is highly expressed in the nucleus accumbens of some pair-bonded species, suggesting that OT may help to encode social reward through modulation of the mesolimbic dopamine pathway (Insel and Shapiro, 1992; Lim and Young, 2006).

Research on the role of OT in HAI has been conducted by measuring OT release during interactions between humans and dogs, and evaluating the effects of exogenous OT administration on dog behavior in this context. Studies of endogenous OT have revealed increases in blood or urinary OT concentrations in both humans and dogs following affiliative interaction in dog-human dyads (Miller et al., 2009; Nagasawa et al., 2009; Handlin et al., 2011; Rehn et al., 2014; Nagasawa et al., 2015). Additionally, preliminary evidence suggests that dogs bred for friendly and non-aggressive temperaments are characterized by high levels of plasma OT, relative to pet dogs (MacLean et al., 2017b). Studies using intranasal OT administration in dogs suggest that exogenous OT can facilitate social play (Romero et al., 2015), and promote bonding with both conspecifics and humans (Romero et al., 2014). Additionally, recent studies have demonstrated that exogenous OT can improve dogs' sensitivity to human communication (Oliva et al., 2015; Macchitella et al., 2016). Thus, a rapidly growing body of research suggests that OT pathways may be centrally involved in affiliative forms of HAI (Beetz et al., 2012; MacLean and Hare, 2015; Carter and Porges, 2016).

We are not aware of any studies investigating AVP in the context of HAI, however, many of the effects of OT are also critically dependent on AVP (Carter, 1998; Landgraf and Neumann, 2004). Although AVP plays important roles in selective sociality, particularly among males (Caldwell et al., 2008), its effects are often antagonistic to those of OT. For example, many of the social effects of OT during HAI may be facilitated by OT's attenuation of sympathetic arousal (Kis et al., 2014; Buttner, 2016) through actions in the hypothalamus (Dabrowska et al., 2011), and on the vagus nerve (Porges, 2003, 2007, 2011). In contrast, AVP activates the hypothalamicpituitary-adrenal (HPA) axis, and is more strongly linked to anxiety and aggression (Coccaro et al., 1998; Neumann and Landgraf, 2012). We are aware of two studies investigating relationships between AVP and behavior in dogs, both of which revealed positive associations with anxiety or aggression (Hydbring-Sandberg et al., 2004; MacLean et al., 2017b). Given its structural and functional relationships with OT, we expect that AVP may also regulate or respond to aspects of HAI, although these effects are likely to differ from those for OT.

To date, all studies of OT and AVP in dogs engaged in HAI have quantified peptide concentrations in blood or urine samples. Although urine sampling can be performed noninvasively, it yields poor temporal resolution and characterizes long periods of peptidergic activity. In contrast, blood sampling can capture acute changes in peptide release, but is an invasive procedure that may induce stress and acute pain, and is not well suited to many of the contexts in which HAI studies are conducted. Additionally, OT and AVP rapidly bind to other molecules in blood (and likely urine) which can lead to matrix interference in assays, or erroneously low estimates of peptide concentrations (Martin and Carter, 2013; Brandtzaeg et al., 2016). We recently validated methods for quantifying OT and AVP in dog saliva samples, which can be collected noninvasively, and at precise time points during HAI. Enzyme-linked immunosorbent assays (ELISAs) yielded good parallelism and accuracy, and did not require an extraction procedure. Lastly, we previously measured salivary OT in nursing dams and detected an acute rise in OT associated with milk letdown, providing an initial biological validation of this measure (MacLean et al., 2017a).

In the current studies, we evaluated both plasma and salivary OT and AVP concentrations in dogs, before and after affiliative interaction with a human, or in a control condition. Because the time course of salivary OT/AVP release during HAI was unknown, we first conducted a short pilot study to identify time points associated with changes in salivary OT/AVP concentrations during HAI. We then conducted an experiment with dogs assigned to an HAI or control condition, and assessed OT/AVP changes over time in both groups, and as a function of behavior during the test period. Based on the studies described above, we hypothesized that dogs in the HAI condition would exhibit increases in OT across the study period, and that any such changes would be larger in the HAI group than the control group. We also hypothesized that within the HAI group, changes in OT would be predicted by the extent of affiliative behavior between the human and the dog during the study. Because no studies have investigated effects of HAI on AVP, we had no specific hypotheses for the nature of this response. However, given that AVP also plays important roles in social emotions and behavior, we expected that dogs in the HAI group would exhibit changes in AVP that differed from those of dogs in the control group.

\section{PILOT STUDY}

Prior to the main HAI study, we conducted a short pilot study with 10 dogs to identify the optimal sampling periods for 
detecting changes in salivary OT and AVP. Based on our studies with nursing dams (MacLean et al., 2017a), and recent studies measuring salivary OT in humans (de Jong et al., 2015), we expected changes in saliva to be rapid. Therefore, we assessed both plasma and salivary OT and AVP at 5 and 10 min following the start of HAI. In both the pilot study and Experiment 1, we opted to collect both blood and saliva, despite the possibility that blood draws may impose a mild stressor with potential to influence hormonal changes detected saliva. We adopted this strategy because no studies have measured salivary OT/AVP in dogs during HAI, and consequently any effects (or lack thereof) in saliva would be challenging to interpret without knowledge of how blood concentrations had changed across the same period.

\section{Method}

\section{Subjects}

We tested dog subjects from the breeding colony at Canine Companions for Independence (CCI) in Santa Rosa, CA, United States. The pilot sample included 10 dogs (eight female, two male, four Labrador retrievers, six Labrador retriever $\times$ golden retriever crosses, mean age $=1.7$ years (range $=1.6-1.8$ years)). All dogs were pair-housed in indooroutdoor kennels with ad libitum access to water and daily access to large outdoor play yards. Subjects were tested in a quiet room inside a familiar building on CCI's campus. Client consent was obtained for participation of all dogs and all animal procedures were approved by the Duke University IACUC (protocol \#A13811-06).

\section{Procedure}

Prior to the test, each dog was allowed to rest quietly in a crate (outside the test room) for $30 \mathrm{~min}$. The dog was then taken to a nearby room and we collected a baseline blood and saliva sample. Saliva samples were collected using the Salimetrics Children's swab as described in MacLean et al. (2017a). Blood samples were collected from the cephalic vein into vacutainers $(3 \mathrm{~mL})$ containing ethylenediaminetetraacetic acid (EDTA). Blood and saliva samples were collected concurrently to minimize the time required for these procedures. Specifically, at the start of the collection period, one experimenter placed the swabs between the dog's cheek and mandibular teeth and gently held the dog's mouth closed while the second experimenter performed the blood collection. All samples were immediately placed on ice after collection.

Following this initial sample, dogs were allowed to rest in a crate inside the test room for $5 \mathrm{~min}$ prior to the start of the behavioral interaction. After $5 \mathrm{~min}$, dogs were released from the crate and allowed to interact freely with the experimenter. The experimenter attempted to engage the dog in friendly interaction, including gently petting the dog, and speaking to the dog in a friendly tone while making eye contact. However, the experimenter allowed the dog to lead these interactions, and dogs were always free to disengage and move away from the experimenter at any point during the interaction. If subjects attempted to engage the experimenter in play (e.g., performing a play bow, chasing, or nuzzling the experimenter), the experimenter engaged with the dog in these more active forms of interaction. After 5 min of HAI we collected a second blood and saliva sample from the dog, and immediately resumed HAI for another $5 \mathrm{~min}$.

The final blood and saliva samples were collected $10 \mathrm{~min}$ after the start of HAI and dogs received a food reward at the conclusion of this period (following the final saliva collection). The second and third blood samples were collected from (1) the cephalic vein on the opposite forelimb from the initial draw, and (2) the jugular vein, in order to avoid repeated needle punctures in the same location. We did not use a catheter for repeated collections from the same site because pilot tests revealed that catheters did not reliably maintain access to the vein when dogs were allowed to move freely between collection periods, and the procedures required to position, wrap, and access the catheter repeatedly were deemed more likely to cause discomfort for the dog than single collections from different sites. All biological samples were immediately frozen at $-20^{\circ} \mathrm{C}$.

\section{Hormone Analysis}

All samples were analyzed by enzyme-linked immunoassay (ELISA) following protocols previously validated in our laboratory (MacLean et al., 2017a,b). OT samples were measured using a commercially available kit from Cayman Chemical (Item \#500440) and AVP samples were measured using a commercially available kit from Enzo Life Sciences (ADI-900-017A). Saliva samples were not extracted based on the results of validation studies (MacLean et al., 2017a) but plasma samples were processed using solid phase extraction (to isolate free peptide concentrations) with the protocols described in MacLean et al. (2017b).

\section{Statistical Analysis}

All statistical analyses were performed in the $\mathrm{R}$ programming environment for statistical computing (R Core Team, 2017). For each matrix (saliva, plasma) and hormone (OT, AVP), we used linear mixed models to predict the log transformed hormone concentration as a function of a fixed effect of time (baseline, $+5 \mathrm{~min},+10 \mathrm{~min}$ ) and a random effect of subject ID. We used planned Dunnett contrasts to assess mean differences between hormone concentrations at baseline and $+5 \mathrm{~min}$, and between baseline and $+10 \mathrm{~min}$.

\section{Results and Discussion}

Table 1 shows the results of linear mixed models predicting changes in hormone concentrations as a function of time. We inspected the data for time points associated with the largest deviations from baseline values.

Salivary OT increased slightly from baseline to $+5 \mathrm{~min}$, with a further increase at +10 min that was marginally different from baseline ( $p=0.05$; Table 1). Changes in plasma OT and both salivary and plasma AVP were minimal, with no clear deviations from baseline. Therefore, we identified the +10 -min measure as the most likely to show an HAI related effect in salivary OT, and collected samples at this time point in Experiment 1. 
TABLE 1 | Pilot study results.

\begin{tabular}{|c|c|c|c|c|c|c|}
\hline & \multicolumn{3}{|c|}{ T5-T0 } & \multicolumn{3}{|c|}{ T10-T0 } \\
\hline & $\beta$ & $S E$ & $p$ & $\beta$ & $S E$ & $p$ \\
\hline Salivary OT & 0.14 & 0.17 & 0.40 & 0.33 & 0.17 & 0.05 \\
\hline Plasma OT & 0.03 & 0.04 & 0.48 & 0.01 & 0.04 & 0.83 \\
\hline Salivary AVP & -0.19 & 0.15 & 0.22 & -0.05 & 0.15 & 0.73 \\
\hline Plasma AVP & -0.05 & 0.12 & 0.67 & -0.07 & 0.12 & 0.56 \\
\hline
\end{tabular}

Baseline (TO) was used as the reference value and the T5-TO and T10-TO Dunnett contrasts show the estimates, and standard errors of estimates, for changes in

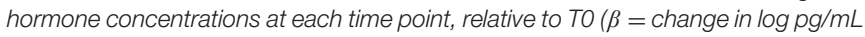
from TO).

TABLE 2 | Subject demographics by condition for Experiment 1.

\begin{tabular}{lccc}
\hline Condition & Breed & Female & Male \\
\hline HAl & LAB & 5 & 1 \\
\multirow{2}{*}{ Control } & LGX & 6 & 7 \\
& LAB & 4 & 1 \\
& LGX & 8 & 6
\end{tabular}

HAl, human-animal interaction; $L A B$, labrador retriever; $L G X$, labrador $\times$ golden retriever cross.

\section{EXPERIMENT 1}

\section{Method \\ Subjects}

We tested 38 dogs (11 Labrador retrievers, 27 Labrador retriever $\times$ golden retriever crosses, 23 female, 15 male, mean age $=1.8$ years, range $=1.6-2.2$ years). All subjects were assistance dogs in training at Canine Companions for Independence and were housed and cared for as described above. Half of the subjects were assigned to the HAI condition, and half were assigned to the control condition (see below). Groups were matched closely based on sex and breed (Table 2 ).

\section{Procedure}

The HAI procedure was identical to that in the pilot study with the exception that we collected blood and saliva samples at only one time point $(+10 \mathrm{~min})$ after the baseline measure, based on preliminary data suggesting the greatest changes in salivary OT at this time. Therefore, the experimenter and dog had 10 continuous minutes of interaction prior to the post-test sample collection. In the control condition, dogs were placed inside an exercise pen $(\sim 3 \mathrm{~m} \times 3 \mathrm{~m})$ in the test room. The experimenter remained in the room with the dog but did not interact, speak to, or make eye contact with the dog during the 10-min test period. We opted to use an exercise pen because this structure provided dogs with opportunities to locomote similarly to dogs in the HAI condition, but prevented dogs from physically accessing the experimenter. Additionally, because all subjects were accustomed to resting quietly in novel environments during their training for assistance work, it was unlikely that containment inside the exercise pen would impose a significant stressor. As in the HAI condition, after $10 \mathrm{~min}$ we collected a post-test blood and saliva sample. After the study, dogs in both conditions received a food reward.

\section{Behavioral Coding}

To assess whether specific behaviors or forms of social interaction (SI) were related to individual differences in hormonal response, we coded the duration (s) of several behaviors from video. Prior to coding, we reviewed all videos to determine which behaviors could be meaningfully coded, considering both the prevalence and observability for a range of theoretically relevant measures. Locomotion and postural variables were coded for subjects in both the control and HAI conditions, and consisted of the following: (1) locomotion: time walking, running, or jumping, with the onset of the behavior marked by three consecutive steps (to disambiguate locomotion from minor bodily repositioning), and of the offset of the behavior marked by a lack of movement for $\geq 1 \mathrm{~s}$; (2) upright: time standing, walking, running, or jumping; (3) lying (prone): time lying with abdomen against the floor; (4) lying (supine): time lying on back or side with abdomen exposed; (5) sitting: time with rump on ground and forelegs extended.

For subjects in the HAI condition we also coded the following behaviors relating to interaction with the experimenter: (6) physical contact: time during which any part of the experimenter's body was in physical contact with any part of the dog's body; (7) licking: time in which the dog's tongue was in contact with the experimenter's body; (8) play: time in which the dog engaged in play bows, chasing, or gentle mouthing with the experimenter, with the onset marked by the first occurrence of any of these behaviors, and the offset marked by a period of $\geq 2 \mathrm{~s}$ without the dog engaging in any of these behaviors. A second independent rater scored all behaviors for random sample of $\sim 50 \%$ of observations, and inter-rater reliability was excellent for all measures (Pearson correlation, mean: $R=0.997)$ $(\min =0.993, \max =1.0)$.

\section{Hormonal Analysis}

Plasma and salivary OT and AVP were assayed using the same ELISA kits and protocols employed in the pilot study. Inter-assay coefficients of variation were $11.1 \%$ (salivary OT), $4.2 \%$ (salivary AVP), 11.7\% (plasma OT) and 11.2\% (plasma AVP).

\section{Statistical Analysis}

All statistical analyses were performed in the $\mathrm{R}$ programming environment for statistical computing ( $\mathrm{R}$ Core Team, 2017). To compare changes in OT/AVP across time between conditions, we used linear mixed models with the log transformed OT/AVP concentrations predicted as a function of fixed effects for sex (male, female), time (pre, post), condition (control, HAI), the time $\times$ condition interaction, and a random effect for subject ID. This model was fitted separately with each endocrine measure as the dependent variable. Because we predicted differential effects over time between groups, we conducted planned contrasts from these models assessing the effect of time within condition, and differences between conditions at each time point. Due to the exploratory nature of the study, we used an alpha value of 


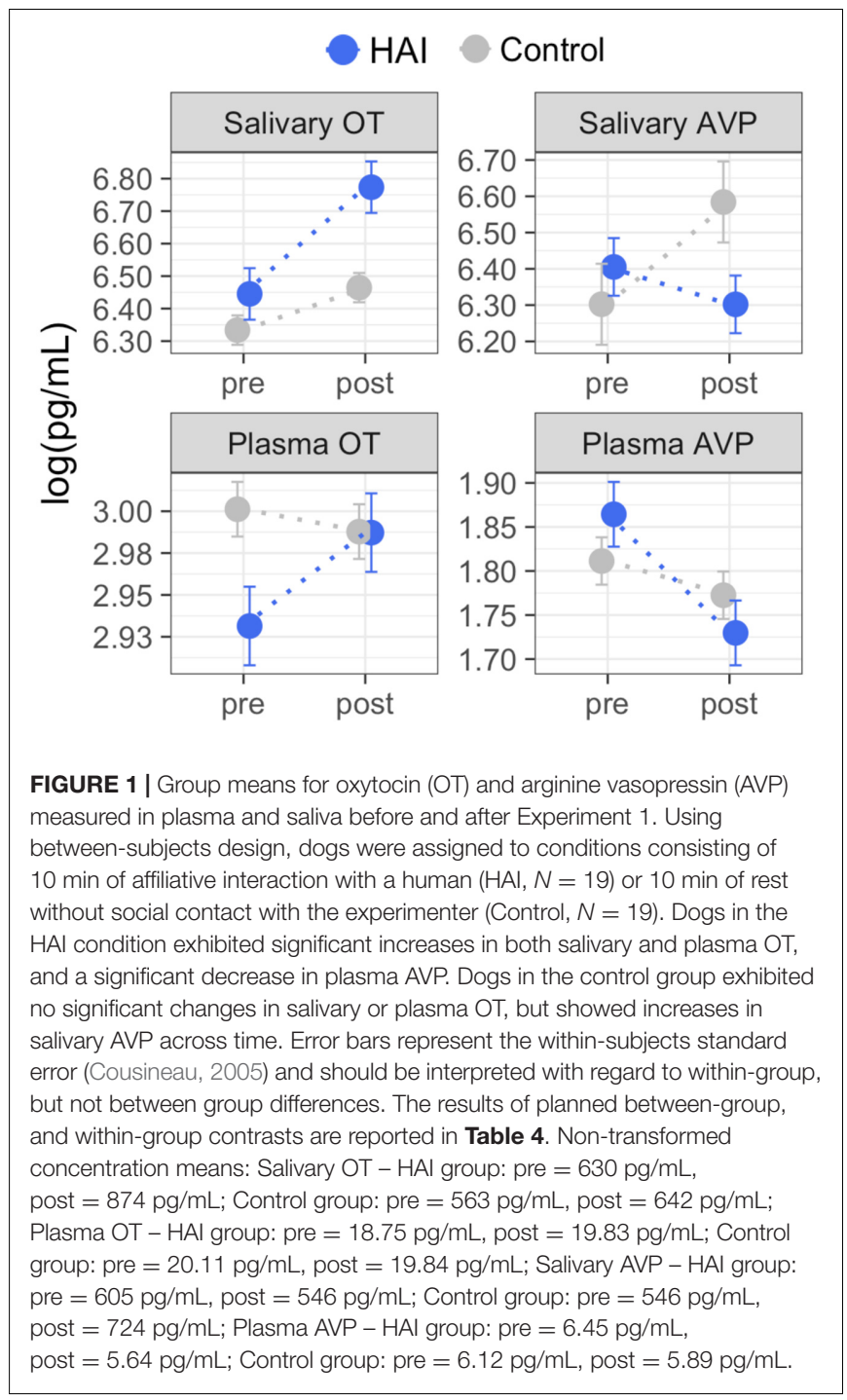

$p \leq 0.05$ for significance testing, without correction for multiple comparisons.

Due to the number of behavioral variables, and substantial correlations between them, we conducted principal components analyses (PCA) to derive a smaller set of behavioral measures for statistical modeling. Because the postural and locomotion variables were coded for subjects in both the control and HAI conditions, we performed a PCA with these measures including data from all subjects. For subjects in the HAI condition, we conducted a second PCA including measures related to SI. The postural variable "lying (supine)" was observed predominantly in conjunction with abdomen petting in the HAI condition and was rarely observed in the control group (median duration in control group $=0$ ). Thus, we omitted this measure from the PCA with variables related to locomotion, and included it in the PCA with variables related to SI. To limit the impact of outliers and skew, all behavioral variables were Yeo-Johnson transformed (Yeo and Johnson, 2000), centered, and scaled prior to fitting the PCA. To determine the number of components to retain, we conducted parallel analysis (Horn, 1965) comparing eigenvalues from the actual data to randomly resampled (with replacement, and dimensions equal to those of the original data) and simulated data (random data from a normal distribution). These analyses suggested retention of two components for both the locomotion and SI PCAs. To assess relationships between these behavioral variables and changes in OT/AVP across the experiment, we fitted linear models predicting the log transformed percent change in OT/AVP concentration as a function of fixed effects for sex (male, female) and scores from the first two principal components. To accommodate negative values in the dependent measure, we first added a constant to all values (the absolute value of the smallest observation +1 ) prior to log transformation. Because the control group did not have component scores for the SI variables, models for this group included only sex and principal component scores for the PCA on locomotion/postural variables. Coefficients for fixed effects were tested with likelihood ratio tests comparing the full model, to nested models with the removal of individual terms.

\section{Results}

\section{Between Group OT/AVP Effects}

Figure 1 shows the mean OT/AVP concentrations across time in the HAI and control groups. Results from the full mixed model for each matrix are shown in Table 3, and the results of planned contrasts comparing OT/AVP concentrations between groups, and assessing within group changes over time, are shown in Table 4.

For salivary OT, planned comparisons revealed that whereas the control and experimental groups had comparable mean salivary OT levels at baseline, dogs in the experimental group had significantly higher post-test salivary OT than controls. Similarly, salivary OT exhibited a significant increase from baseline to posttest in the HAI, but not the control group (Figure 1 and Table 4). For salivary AVP, there were no between group differences at either time point, however, the control group exhibited a significant increase in salivary AVP whereas the HAI group did not (Figure 1 and Table 4).

Plasma OT did not differ between groups at either time point, however, only the HAI group exhibited a modest, but significant, increase in plasma OT over the course of the study (Figure 1 and Table 4). Lastly, there were no group differences in plasma AVP at either time point, however, the HAI group exhibited a significant decrease in plasma AVP across time, whereas the control group did not (Figure 1 and Table 4).

\section{Behavioral Predictors of Changes in OT and AVP}

We retained two components from the PCA including variables related to locomotion and posture (LP), which collectively explained $80 \%$ of variance in these behaviors. Variable loadings from this model are shown in Table 5. The first component (LPPC1, 50\% variance explained) had strong positive loadings for locomotion and upright posture and moderate negative loadings for lying in in a prone position. The second component (LP-PC2, $30 \%$ variance explained) had a strong positive loading for sitting and moderate negative loadings for locomotion, upright posture, and lying (prone). 
TABLE 3 | Results of linear mixed models predicting hormone concentrations as a function of time, condition, sex, and the time by condition interaction.

\begin{tabular}{|c|c|c|c|c|c|c|c|c|c|c|c|c|}
\hline & \multicolumn{6}{|c|}{ Oxytocin } & \multicolumn{6}{|c|}{ Vasopressin } \\
\hline & \multicolumn{3}{|c|}{ Saliva } & \multicolumn{3}{|c|}{ Plasma } & \multicolumn{3}{|c|}{ Saliva } & \multicolumn{3}{|c|}{ Plasma } \\
\hline & $\beta$ & $x^{2}$ & $p$ & $\beta$ & $x^{2}$ & $P$ & $\beta$ & $X^{2}$ & $p$ & $\beta$ & $x^{2}$ & $p$ \\
\hline Time & 0.13 & 1.95 & 0.16 & -0.01 & 0.22 & 0.64 & 0.28 & 4.19 & 0.04 & -0.01 & 0.22 & 0.64 \\
\hline Condition & 0.11 & 0.73 & 0.39 & -0.07 & 0.88 & 0.35 & 0.12 & 0.31 & 0.58 & -0.07 & 0.88 & 0.35 \\
\hline Sex & 0.01 & 0.00 & 0.95 & 0.06 & 0.70 & 0.40 & 0.19 & 0.95 & 0.33 & 0.06 & 0.70 & 0.40 \\
\hline Time $\times$ Condition & 0.20 & 2.28 & 0.13 & 0.07 & 2.93 & 0.09 & -0.39 & 4.01 & 0.05 & 0.07 & 2.93 & 0.09 \\
\hline
\end{tabular}

Planned contrasts from these models are shown in Table 4.

TABLE 4 | Results of planned contrasts between the HAl and control groups at each time point, and across time within groups.

\begin{tabular}{|c|c|c|c|c|c|c|c|c|c|c|c|c|}
\hline & \multicolumn{6}{|c|}{ Group comparison (HAI vs. Control) } & \multicolumn{6}{|c|}{ Within group change (Pre vs. Post) } \\
\hline & \multicolumn{3}{|c|}{ Pre-test } & \multicolumn{3}{|c|}{ Post-test } & \multicolumn{3}{|c|}{ HAI group } & \multicolumn{3}{|c|}{ Control group } \\
\hline & $\beta$ & SE & $p$ & $\beta$ & SE & $p$ & $\beta$ & SE & $p$ & $\beta$ & SE & $p$ \\
\hline Salivary OT & 0.11 & 0.13 & 0.39 & 0.31 & 0.13 & 0.02 & 0.33 & 0.09 & $<0.01$ & 0.13 & 0.09 & 0.16 \\
\hline Salivary AVP & 0.12 & 0.21 & 0.58 & -0.27 & 0.21 & 0.20 & -0.10 & 0.13 & 0.44 & 0.28 & 0.13 & 0.04 \\
\hline Plasma OT & -0.07 & 0.07 & 0.35 & 0.00 & 0.07 & 0.97 & 0.06 & 0.03 & 0.05 & -0.01 & 0.03 & 0.64 \\
\hline Plasma AVP & 0.05 & 0.07 & 0.49 & -0.05 & 0.07 & 0.47 & -0.13 & 0.05 & $<0.01$ & -0.04 & 0.05 & 0.39 \\
\hline
\end{tabular}

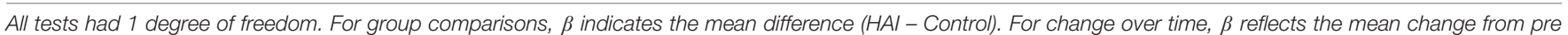
to post.

TABLE 5 | Variable loadings from principal component analyses including variables related to locomotion and posture (all subjects) and social interaction ( $\mathrm{HAl}$ condition only).

\begin{tabular}{llrr}
\hline & & \multicolumn{2}{c}{ Loadings } \\
\cline { 3 - 4 } & Variable & PC1 & PC2 \\
\hline Locomotion/PosturePCA (all subjects) & locomotion & 0.62 & -0.17 \\
& upright & 0.64 & -0.16 \\
& sitting & -0.02 & 0.83 \\
& lying (prone) & -0.44 & -0.50 \\
& physical contact & -0.62 & 0.23 \\
& play & 0.55 & 0.32 \\
& licking & 0.32 & 0.71 \\
& lying (supine) & -0.46 & 0.58 \\
\hline
\end{tabular}

For dogs in the HAI condition, we retained two components from the PCA with variables relating to SI, which collectively explained $81 \%$ of variance in these behaviors. Variable loadings from this model are shown in Table 5. The first component (SIPC1, 53\% variance explained) was loaded positively by play and licking, and negatively by physical contact and lying (supine). The second component (SI-PC2, 28\% variance explained) was loaded positively by all four SI variables (physical contact, play, licking, and lying supine).

Associations between changes in OT/AVP concentrations and behavior during the test are shown in Table 6. In the HAI condition, the percent increase in salivary OT was positively associated with SI-PC2 scores, which reflect longer durations of physical contact, play, licking, and lying (supine) with the abdomen exposed to the experimenter. Despite this positive association with salivary OT, there were no associations between changes in plasma OT concentrations and any of the behavioral variables (Table 6). There were also no associations between changes in salivary AVP and any of the behavioral variables, however, SI-PC2 scores were negatively related to the percent change in plasma AVP. On average, subjects in the HAI group exhibited a $10 \%$ decrease in plasma AVP across the study. However, subjects with SI-PC2 scores in the upper 50th percentile (high levels of affiliative behavior with experimenter) exhibited a larger decrease in plasma AVP (mean change $=-18 \%$, $\mathrm{SEM}=6.89 \%)$ than subjects with SI-PC2 scores in the lower $50^{\text {th }}$ percentile (mean change $=-4 \%$, SEM $=4.87$ ). Within the HAI group, LP-PC2 scores were also negatively associated with the change in plasma AVP. Thus, subjects who engaged in more sitting, and less standing, active locomotion and lying (prone) exhibited larger decreases in plasma AVP across time. Within the control group, there were no significant associations between any of the behavioral measures and changes in peptide concentrations, in saliva or plasma (Table 6).

\section{Discussion}

We assessed changes in dog salivary and plasma OT and AVP in response to HAI, or a control condition. Dogs in the HAI group exhibited increases in both salivary and plasma OT, whereas neither of these effects were observed in the control group. Dogs in the HAI group exhibited a decrease in plasma (but not salivary) AVP across time, whereas dogs in the control group exhibited an increase in salivary AVP, with no significant changes in plasma AVP. Within the HAI group, the extent of the increase in salivary $\mathrm{OT}$, and the decrease in plasma AVP were predicted by the 
TABLE 6 | Associations between changes in hormone concentrations in plasma and saliva and behavior during the test.

\begin{tabular}{|c|c|c|c|c|c|c|c|c|c|c|c|c|c|}
\hline & \multirow[b]{2}{*}{$\begin{array}{l}\text { Matrix / } \\
\text { hormone }\end{array}$} & \multicolumn{3}{|c|}{$\begin{array}{l}\text { Social interaction } \\
\qquad-\mathrm{PCl}\end{array}$} & \multicolumn{3}{|c|}{$\begin{array}{l}\text { Social interaction } \\
\text {-PC2 }\end{array}$} & \multicolumn{3}{|c|}{$\begin{array}{l}\text { Locomotion and Posture } \\
\qquad-\mathrm{PCl}\end{array}$} & \multicolumn{3}{|c|}{$\begin{array}{l}\text { Locomotion and Posture } \\
\qquad \text {-PC2 }\end{array}$} \\
\hline & & $\beta$ & $x^{2}$ & $p$ & $\beta$ & $x^{2}$ & $p$ & $\beta$ & $x^{2}$ & $p$ & $\beta$ & $x^{2}$ & $p$ \\
\hline \multirow[t]{4}{*}{ HAl group } & Salivary OT & -0.60 & 1.55 & 0.21 & 0.61 & 4.37 & 0.04 & 0.72 & 2.70 & 0.10 & 0.22 & 0.60 & 0.44 \\
\hline & Salivary AVP & -0.02 & 0.00 & 0.97 & 0.16 & 0.22 & 0.64 & 0.10 & 0.04 & 0.84 & 0.18 & 0.26 & 0.61 \\
\hline & Plasma OT & -0.26 & 0.44 & 0.51 & 0.04 & 0.04 & 0.84 & 0.25 & 0.51 & 0.48 & 0.18 & 0.60 & 0.44 \\
\hline & Plasma AVP & -0.15 & 0.22 & 0.64 & -0.49 & 6.15 & 0.01 & 0.21 & 0.52 & 0.47 & -0.40 & 3.96 & 0.05 \\
\hline \multirow[t]{4}{*}{ Control group } & Salivary OT & - & - & - & - & - & - & -0.06 & 0.07 & 0.80 & -0.05 & 0.03 & 0.85 \\
\hline & Salivary AVP & - & - & - & - & - & - & -0.36 & 1.80 & 0.18 & 0.20 & 0.35 & 0.56 \\
\hline & Plasma OT & - & - & - & - & - & - & 0.12 & 0.89 & 0.35 & 0.13 & 0.58 & 0.45 \\
\hline & Plasma AVP & - & - & - & - & - & - & 0.13 & 2.49 & 0.12 & -0.04 & 0.15 & 0.70 \\
\hline
\end{tabular}

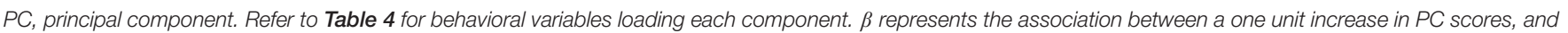
the log transformed percent change in hormone concentrations across time.

degree of affiliative contact between the dog and experimenter. Therefore, both the between and within group differences are most likely attributable to the SIs between the experimenter and dog, and are unlikely to be accounted for by alternative explanations such as a stress response following the initial sample collection.

Although we observed a significant increase in both plasma and salivary OT, the effect was much more pronounced in saliva, echoing the findings of our pilot study. There are at least three reasonable explanations for this finding. First, plasma OT responses can occur extremely rapidly, and may be most evident within $90 \mathrm{~s}$ of a triggering event (Jonas et al., 2009). In contrast, time series analyses suggest a delay in the transfer of hormones from plasma to saliva, and hormonal peaks in saliva have been documented to occur $\sim 10$ min after those in blood (Hernandez et al., 2014). Although the timing, and specific mechanisms through which OT and AVP reach saliva in dogs are unknown, it is probable that changes in salivary concentrations lag behind those in plasma, despite the fact that changes in salivary OT occur quickly relative to other salivary hormones (de Jong et al., 2015). Because this study was designed predominantly for salivary measures, we collected samples at the time point characterized by the highest salivary OT levels in our pilot study. Therefore, is it possible that the largest changes in plasma OT occurred quickly, as has been documented in previous studies (Handlin et al., 2011). Nonetheless, we detected a small but significant increase in plasma OT within the HAI group, suggesting similar effects in both blood and saliva.

Second, whereas OT can be measured without interference in non-extracted dog saliva, other components of dog plasma interfere with OT ELISAs, necessitating additional preparatory procedures such as solid phase extraction for the measurement of free OT (MacLean et al., 2017b). Although extraction procedures can eliminate interfering substances (e.g., albumin proteins), they are often characterized by poor recovery of the target analyte, and thus may eliminate 'signal' as well 'noise.' Unpublished data from our laboratory suggest that common extraction procedures fail to recover substantial amounts of free OT in plasma, despite performing well with kit standards. Therefore, in addition to benefits related to welfare, by virtue of not requiring extraction procedures, salivary measures of OT also have methodological advantages relative to blood sampling.

Third, it has recently been demonstrated that OT rapidly binds to other molecules in plasma, and bound OT is likely to evade detection (Brandtzaeg et al., 2016). Although the binding properties of OT in saliva are unknown, it is possible that OT remains free - and consequently detectable - in saliva, more so than in plasma. This possibility is supported by the high concentrations of OT that we observed in saliva relative to plasma, an effect that persists even with solid phase extraction of dog saliva samples (MacLean et al., 2017a).

In addition to these effects on OT, dogs in the HAI condition also exhibited a decrease in plasma AVP, whereas dogs in the control group exhibited an increase in salivary AVP. Because AVP can activate the HPA axis (Scott and Dinan, 1998; Aguilera and Rabadan-Diehl, 2000), one plausible explanation is that these differences reflect a short-term reduction in stress reactivity in the HAI group, and increased stress reactivity in the control group. This possibility is consistent with many other studies documenting that positive SIs can buffer stress responses (DeVries et al., 2003) - including interspecies interactions (Schöberl et al., 2016) - whereas social isolation can have the opposite effect. Although dogs in the control group remained in the same room with the experimenter, they were physically separated from him, and this separation may have imposed a mild psychological stressor. Despite this possibility, we observed minimal behavioral indications of stress or anxiety in this population of dogs, who are accustomed to resting in novel environments without physical contact from humans as a part of their training for assistance work. A second possibility is that the initial blood and saliva collection imposed an acute stressor in both groups, but that this event was socially buffered by OT in the HAI condition. The latter possibility is consistent with many studies documenting OT's ability to supress HPA activity in response to a stressor (Carter and Altemus, 1997). Both possibilities invoke differential stress responses between the two groups, but differ regarding the hypothesized cause of this stress. In the former scenario, 
physical separation from the experimenter acts as a mild stressor for subjects in the control, but not the HAI condition. In the latter scenario, both groups are exposed to the same mild stressor (i.e., initial blood draw), but stress is buffered more effectively in the HAI group. Although we cannot distinguish between these, and other explanations at present, future studies will benefit by exploring the dynamics between OT, AVP and HPA activity in the context of HAI. Additionally, the differential changes in AVP between groups were observed in different matrices (increased salivary AVP in the control group, decreased plasma AVP in the HAI group), and the explanation for this phenomenon remains unknown. Given that AVP is released in response to both acute and chronic stress, it will be important to investigate the significance of both plasma and salivary vasopressin in future studies explicitly focusing on dogs' biological responses to potentially stressful events.

Lastly, in addition to the between group differences that we observed, within the HAI group the extent of the increase in salivary OT, and decrease in plasma AVP, were predicted the nature of interactions between the dog and experimenter. Specifically, dogs who engaged in higher levels of physical contact, play, licking and lying in a supine position with the experimenter exhibited the largest increases in OT, and decreases in AVP. Thus, as in previous studies, changes in OT and AVP concentrations depended not only on human contact, but also on the nature of these interactions (Rehn et al., 2014; Nagasawa et al., 2015).

Collectively, our results corroborate previous findings suggesting that OT responds dynamically to HAI, and provide the first data on HAI's effects on AVP in dogs. Notably, although HAI-related effects on OT have also been observed in humans, we are not aware of any studies examining AVP in humans in

\section{REFERENCES}

Aguilera, G., and Rabadan-Diehl, C. (2000). Vasopressinergic regulation of the hypothalamic-pituitary-adrenal axis: implications for stress adaptation. Regul. Pept. 96, 23-29. doi: 10.1016/S0167-0115(00)00196-8

Beetz, A., Uvnäs-Moberg, K., Julius, H., and Kotrschal, K. (2012). Psychosocial and psychophysiological effects of human-animal interactions: the possible role of oxytocin. Front. Psychol. 3:234. doi:10.3389/fpsyg.2012.0023

Brandtzaeg, O. K., Johnsen, E., Roberg-Larsen, H., Seip, K. F., MacLean, E. L., Gesquiere, L. R., et al. (2016). Proteomics tools reveal startlingly high amounts of oxytocin in plasma and serum. Sci. Rep. 6:31693. doi: 10.1038/srep31693

Buttner, A. P. (2016). Neurobiological underpinnings of dogs' human-like social competence: how interactions between stress response systems and oxytocin mediate dogs' social skills. Neurosci. Biobehav. Rev. 71, 198-214. doi: 10.1016/j. neubiorev.2016.08.029

Caldwell, H., and Young, W. III. (2006). Oxytocin and Vasopressin: Genetics and Behavioral Implications Handbook of Neurochemistry and Molecular Neurobiology. Berlin: Springer, 573-607. doi: 10.1007/978-0-387-30381-9_25

Caldwell, H. K., Lee, H.-J., Macbeth, A. H., and Young, W. S. III. (2008). Vasopressin: behavioral roles of an "original" neuropeptide. Prog. Neurobiol. 84, 1-24. doi: $10.1016 /$ j.pneurobio.2007.10.007

Carter, C., and Altemus, M. (1997). Integrative functions of lactational hormones in social behavior and stress management. Ann. N. Y. Acad. Sci. 807, 164-174. doi: 10.1111/j.1749-6632.1997.tb51918.x

Carter, C., Williams, J. R., Witt, D. M., and Insel, T. R. (1992). Oxytocin and social bonding. Ann. N. Y. Acad. Sci. 652, 204-211. doi: 10.1111/j.1749-6632.1992. tb34356.x the context of interaction with animals. Given that we observed significant decreases in dogs' plasma AVP concentrations following HAI, we expect that future studies will benefit by incorporating this measure in humans as well. Collectively, these studies suggest that salivary measures of OT and AVP provide non-invasive biomarkers which respond to aspects of affiliative social behavior, and provide researchers with a new set of tools for exploring the roles of OT and AVP in the biology of HAI.

\section{AUTHOR CONTRIBUTIONS}

EM, NG, KL, and CSC designed the experiments. EM and KL collected the data. EM performed immunoassays and analyzed the data. EM, LG, NG, KL, WLM, and CSC wrote the paper.

\section{FUNDING}

We gratefully acknowledge support from the WALTHAM ${ }^{\circledR}$ Centre for Pet Nutrition, which funded this research, and the Stanton Foundation, for a Next Generation Canine Research Fellowship to EM.

\section{ACKNOWLEDGMENTS}

We are grateful to Brenda Kennedy and staff at Canine Companions for Independence for their help with this research. We thank Hossein Nazarloo, Martina Heer, and Taichi Inui for helpful conversations, and Susan Alberts for allowing us to work her laboratory.

Carter, C. S. (1998). Neuroendocrine perspectives on social attachment and love. Psychoneuroendocrinology 23, 779-818. doi: 10.1016/S0306-4530(98) 00055-9

Carter, C. S., Grippo, A. J., Pournajafi-Nazarloo, H., Ruscio, M. G., and Porges, S. W. (2008). Oxytocin, vasopressin and sociality. Prog. Brain Res. 170, 331-336. doi: 10.1016/S0079-6123(08)00427-5

Carter, C. S., and Porges, S. W. (2016). "Neural mechanisms underlying humananimal interaction: an evolutionary perspective," in The Social Neuroscience of Human-animal Interaction, eds L. S. Freund, S. McCune, L. Esposito, N. R. Gee, and P. McCardle (Washington, DC: American Psychological Association), 89-105. doi: 10.1037/14856-006

Coccaro, E. F., Kavoussi, R. J., Hauger, R. L., Cooper, T. B., and Ferris, C. F. (1998). Cerebrospinal fluid vasopressin levels correlates with aggression and serotonin function in personality-disordered subjects. Arch. Gen. Psychiatry 55, 708-714. doi: 10.1001/archpsyc.55.8.708

Cousineau, D. (2005). Confidence intervals in within-subject designs: a simpler solution to Loftus and Masson's method. Tutor. Quant. Methods Psychol. 1, 42-45. doi: 10.20982/tqmp.01.1.p042

Dabrowska, J., Hazra, R., Ahern, T. H., Guo, J.-D., McDonald, A. J., Mascagni, F., et al. (2011). Neuroanatomical evidence for reciprocal regulation of the corticotrophin-releasing factor and oxytocin systems in the hypothalamus and the bed nucleus of the stria terminalis of the rat: implications for balancing stress and affect. Psychoneuroendocrinology 36, 1312-1326. doi: 10.1016/j. psyneuen.2011.03.003

de Jong, T. R., Menon, R., Bludau, A., Grund, T., Biermeier, V., Klampfl, S. M., et al. (2015). Salivary oxytocin concentrations in response to running, sexual selfstimulation, breastfeeding and the TSST: the Regensburg Oxytocin Challenge 
(ROC) study. Psychoneuroendocrinology 62, 381-388. doi: 10.1016/j.psyneuen. 2015.08.027

DeVries, A. C., Glasper, E. R., and Detillion, C. E. (2003). Social modulation of stress responses. Physiol. Behav. 79, 399-407. doi: 10.1016/S0031-9384(03) 00152-5

Handlin, L., Hydbring-Sandberg, E., Nilsson, A., Ejdeback, M., Jansson, A., and Uvnas-Moberg, K. (2011). Short-term interaction between dogs and their owners: effects on oxytocin, cortisol, insulin and heart RateAn exploratory study. Anthrozoos 24, 301-315. doi: 10.2752/175303711X1304591486 5385

Hernandez, C. E., Thierfelder, T., Svennersten-Sjaunja, K., Berg, C., Orihuela, A., and Lidfors, L. (2014). Time lag between peak concentrations of plasma and salivary cortisol following a stressful procedure in dairy cattle. Acta Vet. Scand. 56:61. doi: 10.1186/s13028-014-0061-3

Horn, J. L. (1965). A rationale and test for the number of factors in factor analysis. Psychometrika 30, 179-185. doi: 10.1007/BF0228 9447

Hydbring-Sandberg, E., von Walter, L. W., Hoglund, K., Svartberg, K., Swenson, L., and Forkman, B. (2004). Physiological reactions to fear provocation in dogs. J. Endocrinol. 180, 439-448. doi: 10.1677/joe.0. 1800439

Insel, T. R., and Shapiro, L. E. (1992). Oxytocin receptor distribution reflects social organization in monogamous and polygamous voles. Proc. Natl. Acad. Sci. U.S.A. 89, 5981-5985. doi: 10.1073/pnas.89.13.5981

Jonas, W., Johansson, L., Nissen, E., Ejdebäck, M., Ransjö-Arvidson, A., and UvnäsMoberg, K. (2009). Effects of intrapartum oxytocin administration and epidural analgesia on the concentration of plasma oxytocin and prolactin, in response to suckling during the second day postpartum. Breastfeed. Med. 4, 71-82. doi: 10.1089/bfm.2008.0002

Kis, A., Kanizsár, O., Gácsi, M., and Topál, J. (2014). Intranasally administered oxytocin decreases heart rate and increases heart rate variability in dogs. J. Vet. Behav. Clin. Appl. Res. 9:e15. doi: 10.1016/j.jveb.2014.09.050

Landgraf, R., and Neumann, I. D. (2004). Vasopressin and oxytocin release within the brain: a dynamic concept of multiple and variable modes of neuropeptide communication. Front. Neuroendocrinol. 25, 150-176. doi: 10.1016/j.yfrne. 2004.05.001

Lim, M. M., and Young, L. J. (2006). Neuropeptidergic regulation of affiliative behavior and social bonding in animals. Horm. Behav. 50, 506-517. doi: 10 . 1016/j.yhbeh.2006.06.028

Macchitella, L., Stegagno, T., Giaconella, R., di Sorrentino, E. P., Schino, G., and Addessi, E. (2016). Oxytocin improves the ability of dogs to follow informative pointing: a neuroemotional hypothesis. Rend. Lincei 28, 105-115. doi: 10.1007/ s12210-016-0579-6

MacLean, E., Gesquiere, L., Gee, N., Levy, K., Martin, W. L., and Carter, C. S. (2017a). Validation of salivary oxytocin and vasopressin as biomarkers in domestic dogs. J. Neurosci. Methods. doi: 10.1016/j.jneumeth.2017.08.033 [Epub ahead of print].

MacLean, E., Gesquiere, L., Gruen, M., Sherman, B., Martin, W. L., and Carter, C. S. (2017b). Endogenous oxytocin, vasopressin and aggression in domestic dogs. Front. Psychol. 8:1613. doi: 10.3389/fpsyg.2017.01613

MacLean, E. L., and Hare, B. (2015). Dogs hijack the human bonding pathway. Science 348, 280-281. doi: 10.1126/science.aab1200

Martin, W., and Carter, C. S. (2013). Oxytocin and vasopressin are sequestered in plasma. Paper Presented at the 10th World Congress on Neurohypophysial Hormones, Bristol.

Miller, S. C., Kennedy, C., DeVoe, D., Hickey, M., Nelson, T., and Kogan, L. (2009). An examination of changes in oxytocin levels in men and women before and after interaction with a bonded dog. Anthrozoos 22, 31-42. doi: 10.2752/175303708X390455
Nagasawa, M., Kikusui, T., Onaka, T., and Ohta, M. (2009). Dog's gaze at its owner increases owner's urinary oxytocin during social interaction. Horm. Behav. 55, 434-441. doi: 10.1016/j.yhbeh.2008.12.002

Nagasawa, M., Mitsui, S., En, S., Ohtani, N., Ohta, M., Sakuma, Y., et al. (2015). Oxytocin-gaze positive loop and the coevolution of human-dog bonds. Science 348, 333-336. doi: 10.1126/science.1261022

Neumann, I. D., and Landgraf, R. (2012). Balance of brain oxytocin and vasopressin: implications for anxiety, depression, and social behaviors. Trends Neurosci. 35, 649-659. doi: 10.1016/j.tins.2012.08.004

Oliva, J., Rault, J.-L., Appleton, B., and Lill, A. (2015). Oxytocin enhances the appropriate use of human social cues by the domestic dog (Canis familiaris) in an object choice task. Anim. Cogn. 18, 991. doi: 10.1007/s10071-0150870-4

Pedersen, C. A., and Prange, A. J. (1979). Induction of maternal behavior in virgin rats after intracerebroventricular administration of oxytocin. Proc. Natl. Acad. Sci. U.S.A. 76, 6661-6665. doi: 10.1073/pnas.76.12.6661

Porges, S. W. (2003). The polyvagal theory: phylogenetic contributions to social behavior. Physiol. Behav. 79, 503-513. doi: 10.1016/S0031-9384(03)00156-2

Porges, S. W. (2007). The polyvagal perspective. Biol. Psychol. 74, 116-143. doi: 10.1016/j.biopsycho.2006.06.009

Porges, S. W. (2011). The Polyvagal Theory: Neurophysiological Foundations of Emotions, Attachment, Communication, and Self-regulation (Norton Series on Interpersonal Neurobiology). New York City, NY: W. W. Norton \& Company.

R Core Team (2017). R: A Language and Environment for Statistical Computing. Vienna: R Foundation for Statistical Computing.

Rehn, T., Handlin, L., Uvnäs-Moberg, K., and Keeling, L. J. (2014). Dogs' endocrine and behavioural responses at reunion are affected by how the human initiates contact. Physiol. Behav. 124, 45-53. doi: 10.1016/j.physbeh.2013.10.009

Romero, T., Nagasawa, M., Mogi, K., Hasegawa, T., and Kikusui, T. (2014). Oxytocin promotes social bonding in dogs. Proc. Natl. Acad. Sci. U.S.A. 111, 9085-9090. doi: 10.1073/pnas.1322868111

Romero, T., Nagasawa, M., Mogi, K., Hasegawa, T., and Kikusui, T. (2015). Intranasal administration of oxytocin promotes social play in domestic dogs. Commun. Integr. Biol. 8:e1017157. doi: 10.1080/19420889.2015.1017157

Schöberl, I., Beetz, A., Solomon, J., Wedl, M., Gee, N., and Kotrschal, K. (2016). Social factors influencing cortisol modulation in dogs during a strange situation procedure. J. Vet. Behav. Clin. Appl. Res. 11, 77-85. doi: 10.1016/j.jveb.2015. 09.007

Scott, L. V., and Dinan, T. G. (1998). Vasopressin and the regulation of hypothalamic-pituitary-adrenal axis function: implications for the pathophysiology of depression. Life Sci. 62, 1985-1998. doi: 10.1016/S00243205(98)00027-7

Yeo, I.-K., and Johnson, R. A. (2000). A new family of power transformations to improve normality or symmetry. Biometrika 87, 954-959. doi: 10.1093/biomet/ 87.4.954

Conflict of Interest Statement: The authors declare that this research was funded by the WALTHAM ${ }^{\circledR}$ Centre for Pet Nutrition (WCPN) which employed author NG, whose contribution is outlined in the author contribution statement. WLM was also employed by Martin-Protean LLC. All other authors have no conflicts of interest to declare.

Copyright (C) 2017 MacLean, Gesquiere, Gee, Levy, Martin and Carter. This is an open-access article distributed under the terms of the Creative Commons Attribution License (CC BY). The use, distribution or reproduction in other forums is permitted, provided the original author(s) or licensor are credited and that the original publication in this journal is cited, in accordance with accepted academic practice. No use, distribution or reproduction is permitted which does not comply with these terms. 